\title{
Desenvolvimento do Aplicativo "Cuidados Paliativos" para Auxílio na Avaliação e Assistência de Pacientes
}

\author{
Thábata Queiroz Vivas de Sá ${ }^{1}$ \\ Paulo Rodrigues Gomes ${ }^{1}$ \\ Breno Max Horta Melo ${ }^{1}$ \\ Taís Marina de Souza ${ }^{1}$ \\ Laila Carine Ferreira Lodi Junqueira ${ }^{1}$ \\ Munir Murad Junior ${ }^{1}$ \\ Antônio Luiz Pinho Ribeiro ${ }^{1}$
}

${ }^{1}$ Centro de Telessáude do Hospital das Clínicas, Universidade Federal de Minas Gerais, Brasil; E-Mail: thabatasa@gmail.com; prxgomes@gmail.com; brenommelo@gmail.com; taismarinasz@gmail.com; lailacarine@yahoo.com.br; emeiodanado@gmail.com; alpr1963br@gmail.com

\begin{abstract}
Resumo
Introdução: Em pacientes recebendo Cuidados Paliativos (CP), a necessidade de cuidados e a intensidade dos sintomas aumentam gradativamente, associadas à progressão da doença principal. Objetivo: Descrever o desenvolvimento do aplicativo móvel "Cuidados Paliativos", multiplataforma, de apoio ao profissional de saúde que atua em CP. Método: Uma equipe multidisciplinar procedeu com revisão teórica, levantamento dos requisitos de software, análise do cotidiano dos profissionais em CP e desenvolvimento do aplicativo. Resultado: Aplicativo móvel, que permite ao profissional se apropriar de conceitos básicos de CP e de estratégias para comunicação de más notícias e monitorar os principais sintomas e a funcionalidade do paciente. Em junho de 2017, o aplicativo tinha nota 4,8/5,0 no Google Play e mais de 2.400 downloads. Conclusão: O aplicativo obteve expressivo número de downloads logo após sua publicação, êxito que pode evidenciar o alcance atual do tema e impulsiona a expansão do "Cuidados Paliativos" visando incorporar novas funcionalidades.
\end{abstract}

Palavras-chave: controle de sintomas; cuidados paliativos; más notícias; suporte ao tratamento.

\begin{abstract}
Introduction: In patients receiving Palliative Care (PC), the need for care and the symptoms intensity gradually increase, as the main disease progresses. Objective: Describe the development of the multiplatform mobile app "Palliative Care", to support the health professional who works in PC. Method: A multidisciplinary team proceeded with theoretical revision, survey of software requirements, analysis of PC professionals' daily life and app development. Result: A mobile app, which allows professionals acess to basic PC concepts and strategies for communicating bad news and monitoring patients functionality and symptoms. In June 2017, the app had 4.8 / 5.0 rating on Google Play and more than 2,400 downloads. Conclusion: The app obtained a significant number of downloads soon after its publication, a success that can highlight the theme' scope currently and boost the expansion of "Palliative Care" to incorporate new functionalities.
\end{abstract}

Keywords: bad news; palliative care; symptom control; treatment support. 


\section{Introdução}

A Organização Mundial de Saúde (OMS), em revisão de 2002, define Cuidados Paliativos como “Uma abordagem que promove a qualidade de vida de pacientes e seus familiares, que enfrentam doenças que ameaçam a continuidade da vida, através da prevenção e alívio do sofrimento. Requer a identificação precoce, avaliação e tratamento da dor e outros problemas de natureza física, psicossocial e espiritual” (ANCP Brasil, 2009).

Os Cuidados Paliativos são indicados para todos os pacientes com doença ameaçadora da continuidade da vida, por qualquer diagnóstico, com qualquer prognóstico ou idade e a qualquer momento da doença em que eles tenham expectativas ou necessidades não atendidas.

Ao longo da evolução da doença, a intensidade dos Cuidados Paliativos é variável, sendo que o foco e os objetivos vão, progressivamente, transitando de uma ênfase em tratamentos modificadores da doença até abordagens com intenção exclusivamente paliativas, ou seja, de controle de sintomas que impactam na qualidade de vida.

A prática dos Cuidados Paliativos baseia-se no controle impecável dos sintomas de natureza física, psicológica, social e espiritual (Maciel, 2009). Os princípios do controle destes sintomas se baseiam em (Neto, 2006):

- Avaliar antes de tratar

- Explicar as causas dos sintomas

- Não esperar que um doente se queixe

- Adotar uma estratégia terapêutica mista

- Monitorizar os sintomas

- Reavaliar regularmente as medidas terapêuticas

- Cuidar dos detalhes

- Estar disponível

\section{Objetivo}

Este trabalho objetiva descrever o desenvolvimento de um aplicativo móvel, multiplataforma, de referência em Cuidados Paliativos.

\section{Método}

Estudo descritivo sobre o desenvolvimento de um aplicativo móvel para avaliação e assistência aos pacientes que estão em Cuidados Paliativos.

O aplicativo, denominado "Cuidados Paliativos", foi desenvolvido em 2016 e publicado, no Google Play ${ }^{1}$, no dia 02 de fevereiro 2017, pelo Centro de Telessaúde do Hospital das Clínicas da Universidade Federal de Minas Gerais. 0 projeto contou com uma equipe multidisciplinar para levantamento dos requisitos de software, elaboração da fundamentação teórica, análise do cotidiano dos profissionais atuantes na área e melhores práticas de usabilidade e disponibilização das informações.

As tecnologias, Angular JS e lonic, empregadas no desenvolvimento, foram direcionadas para plataforma móvel utilizando ferramentas para a construção de aplicativos híbridos, no intuito de possibilitar que seja utilizado em diferentes sistemas operacionais (Android, IOS e Windows Fone) (Prezotto \& Boniati, 2014). Para as interfaces foram utilizadas as tecnologias: Bootstrap, jQuery e JavaScript. O banco de dados escolhido foi o CouchDB, por permitir a criação e manuseio de dados off-line com sincronismo nativo.

\footnotetext{
${ }^{1}$ Google Inc., "Termos de Serviço Do Google Play."
} 
Para a gestão e planejamento do projeto foi utilizada a metodologia Scrum, considerada uma metodologia ágil, pois propõe a divisão do projeto em ciclos, normalmente, mensais ou semanais, mas não superiores a 30 dias, chamados de sprints. Cada sprint representa uma parte do projeto, previamente definida, a ser desenvolvida no período. Além disso, a metodologia sugere reuniões rápidas e diárias entre os interessados para avaliar o andamento do projeto e as dificuldades encontradas. Desta forma, o processo de desenvolvimento se torna interativo e dinâmico, propiciando mais agilidade e entregas parciais (Ferreira et al., 2005).

Foi realizada uma extensa pesquisa sobre as indicações preconizadas pelos centros de referência em Cuidados Paliativos. Os dados mais recentes demonstram a relevância de capacitar os profissionais de saúde envolvidos no cuidado do paciente em Cuidados Paliativos quanto ao uso das escalas PPS (Palliative Performance Scale) (Maciel \& Carvalho 2009) e ESAS (Edmonton Symptom Assesment System) (Monteiro 2012), que auxiliam na avaliação da performance física do paciente e no manejo de sintomas. A performance física está associada ao prognóstico das doenças oncológicas e o manejo de sintomas está diretamente relacionado à qualidade de vida do paciente e a qualidade da assistência oferecida.

A Escala de Performance Paliativa (PPS) é uma ferramenta utilizada em situações de elegibilidade para os Cuidados Paliativos, pois permite a avaliação da funcionalidade atual do paciente e a compreensão da trajetória de sua doença de base. 0 escore total varia de $100 \%$, que significa máxima atividade funcional, até $0 \%$, indicando morte (Maida et al., 2008).

A escala ESAS, Edmonton Symptom Assesment System, é uma ferramenta utilizada em Cuidados Paliativos para quantificar os principais sintomas apresentados pelo paciente com o objetivo de orientar o melhor tratamento e melhorar a qualidade de vida. É composta por nove sintomas cardinais, sendo eles: dor, cansaço, náuseas, falta de apetite, tristeza, sonolência, dispneia, bem-estar e ansiedade; e um ou mais sintomas que podem ser vivenciados pelo paciente. A escala varia de 0 (zero) a 10 (dez) sendo zero a ausência de sintomas e 10 o sintoma em sua maior intensidade.

Foi evidenciada a importância de se utilizar estratégias pré-definidas para comunicação de más notícias aos pacientes e familiares, sendo uma delas o acrônimo "PREPARED” (Clayer, 2007).

A usabilidade é um importante fator para melhorar a interação do usuário com o aplicativo. Diversos fatores contextuais e ambientais contribuem ou dificultam a utilização do mesmo (Gonçalves, 2014). Segundo a norma ISO/IEC $25010^{2}$, a usabilidade considera aspectos sobre quão fácil um sistema pode ser usado, como aprendizado, operabilidade, estética, entre outros.

Todas as interfaces foram criadas a fim de aperfeiçoar a experiência do usuário e garantir maior usabilidade, fatores fundamentais qualidade e competitividade do aplicativo (Rivero \& Conte, 2014). Para isso, foram utilizados elementos gráficos, com o cuidado de não poluir a interface, mas com o objetivo de apresentar o conteúdo do aplicativo de forma agradável, por meio de cores, gráficos e imagens.

\section{Resultados}

O desenvolvimento do aplicativo resultou em uma ferramenta de apoio aos profissionais de saúde que necessitam avaliar e obter orientações a respeito do estado de saúde do seu paciente em Cuidados Paliativos. O aplicativo consiste em duas funcionalidades teóricas e três funcionalidades práticas, tendo uma funcionalidade em fase final de desenvolvimento. As funcionalidades desenvolvidas e publicadas podem ser vistas na Figura 1.

\footnotetext{
$2_{2}$ International Organization for Standardization, ISO/IEC 25010 - Systems and software engineering - SQuaRE - Software product Quality Requirements and Evaluation -- System and software quality models.
} 


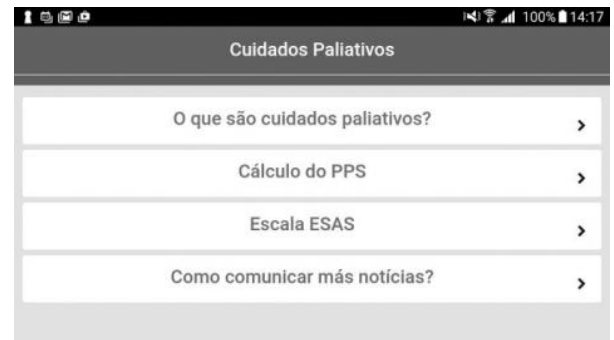

Figura 1. Tela principal do aplicativo

\section{Funcionalidades Teóricas}

As funcionalidades teóricas têm como objetivo apresentar os conceitos que permeiam os Cuidados Paliativos, com base nas melhores referências na literatura.

I. "O que são cuidados paliativos?" - Contém a definição de Cuidados Paliativos e links que podem ser acessados para esclarecimento de dúvidas e outros conceitos específicos.

II. “Como comunicar más notícias?” - Apresenta, de forma objetiva e prática, as orientações para a comunicação de más notícias aos pacientes e familiares. Má notícia é definida como: "Qualquer informação que, de forma adversa, afeta gravemente a visão de um indivíduo sobre o seu futuro" ${ }^{3}$. É utilizado o acrônimo "PREPARED", com detalhamento de cada item, para facilitar a memorização da melhor maneira de executar esta tarefa.

\section{Funcionalidades Práticas}

As funcionalidades práticas são dinâmicas e demandam a interação do usuário com o aplicativo para a obtenção de um resultado ou orientação.

III. Cálculo do PPS - Nesta funcionalidade, os cinco quesitos do PPS são apresentados para serem avaliados e respondidos conforme opções que variam de acordo com a gravidade.

Os quesitos são: 1) Deambulação; 2) Atividade e evidência da doença; 3) Autocuidado; 4) Ingestão e 5) Nível da consciência. A forma de apresentação dos quesitos e suas opções de resposta podem ser vistas na Figura 2.

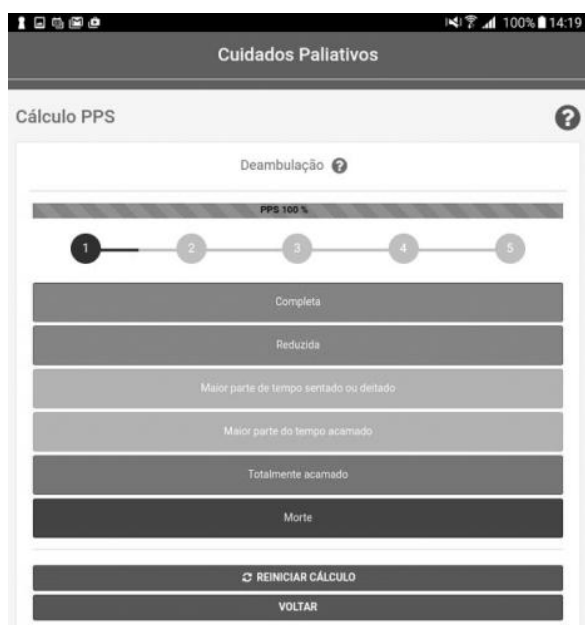

Figura 2. Cálculo do PPS.

\footnotetext{
3 Rede de Teleassistência de Minas Gerais, “Como Comunicar Más Notícias?,” n.d., http://www.telessaude.hc.ufmg.br/wpcontent/uploads/2016/07/8-MA-NOTICIA.V.4_web.pdf.
} 
As cores foram utilizadas para ilustrar a gravidade das opções de respostas, com a finalidade de reforçar o conteúdo descrito e tornar a escolha mais evidente. As cores utilizadas são:

- Verde: indica um nível estável.

- Amarelo: indica um nível transitório, com a necessidade de alguns cuidados.

- Vermelho: indica um nível crítico, com a necessidade de que alguém assuma seus cuidados.

- Cinza: indica a morte do paciente, sem a necessidade de continuidade no preenchimento. 0 escore se torna $0 \%$.

Na parte superior é exibida a pontuação atual (a partir das opções selecionadas) em uma barra de progresso compatível com o sistema de cores descrito acima. As opções de resposta são dispostas conforme a ordem de gravidade e a resposta de um quesito interfere na pontuação do quesito seguinte.

Ao final, é exibida a pontuação total e as orientações a respeito do cuidado preconizado com base no resultado obtido, conforme Tabela 1.

Tabela 1. Orientações do PPS.

\begin{tabular}{|c|c|}
\hline Valor do PPS & Orientação \\
\hline Acima de $70 \%$ & $\begin{array}{l}\text { O PPS entre } 100 \text { e } 70 \% \text { indica que o paciente está estável e necessita de número reduzido } \\
\text { de cuidados e auxílio para a realização das atividades básicas de vida diária. }\end{array}$ \\
\hline Entre $40 \%$ e $60 \%$ & $\begin{array}{l}\text { O paciente com PPS entre } 60 \text { e } 40 \% \text { apresenta uma condição transitória, em que a } \\
\text { demanda por cuidados e auxílio cresce e já existem evidências da progressão da doença. } \\
\text { Nessa condição é necessário considerar os aspectos psicológicos, sociais e econômicos, } \\
\text { bem como as mudanças físicas experimentadas pelo indivíduo, prevenindo prejuízo no } \\
\text { relacionamento entre o paciente e sua família. }\end{array}$ \\
\hline $\begin{array}{l}\text { Abaixo ou igual a } \\
30 \%\end{array}$ & $\begin{array}{l}\text { O paciente com PPS } \leq 30 \% \text { se encontra no estágio avançado, com a capacidade funcional } \\
\text { seriamente comprometida e necessitando que alguém assuma seus cuidados. Nesse caso } \\
\text { é importante preparar o paciente e sua família para a proximidade da morte, com o } \\
\text { objetivo de resolver assuntos pendentes, como por exemplo, elaboração de testamentos, } \\
\text { custódia de filhos, e outros. }\end{array}$ \\
\hline Igual a 0\% & $\begin{array}{l}\text { Os cuidados com o paciente terminaram, portanto é necessário intensificar o suporte aos } \\
\text { familiares e cuidadores envolvidos. }\end{array}$ \\
\hline
\end{tabular}

IV. Escala ESAS - Para propiciar a melhor utilização da funcionalidade, combinamos no aplicativo a escala ESAS com a escala EVA (escala visual analógica) para a avaliação de cada sintoma.

A escala EVA, é originalmente utilizada para avaliação de dor, associa a intensidade do sintoma de forma numérica (de zero a 10) com a escala de gradação de cores (que varia da cor azul, como melhor situação possível, até a cor vermelha, que indica a maior intensidade do sintoma avaliado), com a escala de expressões faciais, que representa a fisionomia do paciente perante o sintoma e com a escala de níveis de sintomas, que agrupa as intensidades em "Leve", "Moderada" e "Intensa".

Os dados podem ser preenchidos pelo paciente, pelo profissional de saúde e/ou pelo cuidador (Monteiro, 2012), conforme Figura 3. 


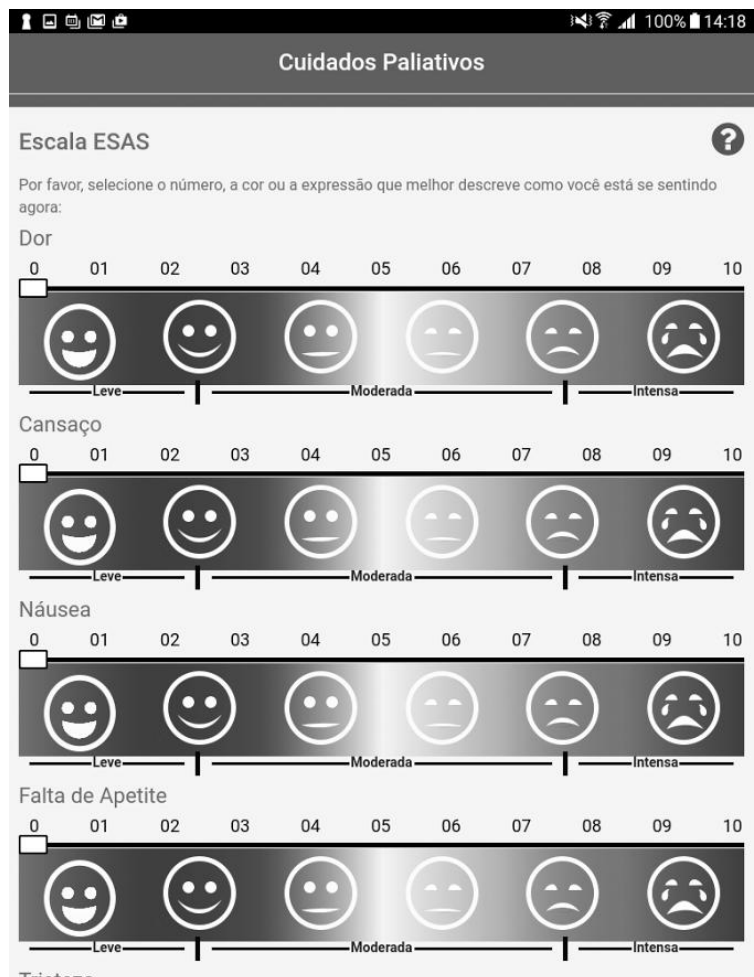

Figura 3. Escala ESAS.

O usuário ao ser apresentado a essa escala pode selecionar a representação com a qual mais se identifica e/ou tem habilidade para utilizar, reduzindo assim o fator escolaridade como uma barreira à estratificação adequada dos sintomas (Thomas, 2011).

O aplicativo possui uma funcionalidade em fase final de desenvolvimento não publicada ainda, para auxiliar profissionais de saúde na identificação de pacientes com necessidade de Cuidados Paliativos.

Esta funcionalidade consiste em um questionário sobre o estado atual de saúde do paciente, a presença de indicadores clínicos (doenças diagnosticadas previamente) e sinais de declínio ou aumento das necessidades de cuidados.

O questionário (Figura 4) foi construído pela equipe científica do projeto, com base em revisão na literatura recente, para oferecer ao usuário facilidade de uso, suporte à decisão e informações completas sobre os Cuidados Paliativos. 


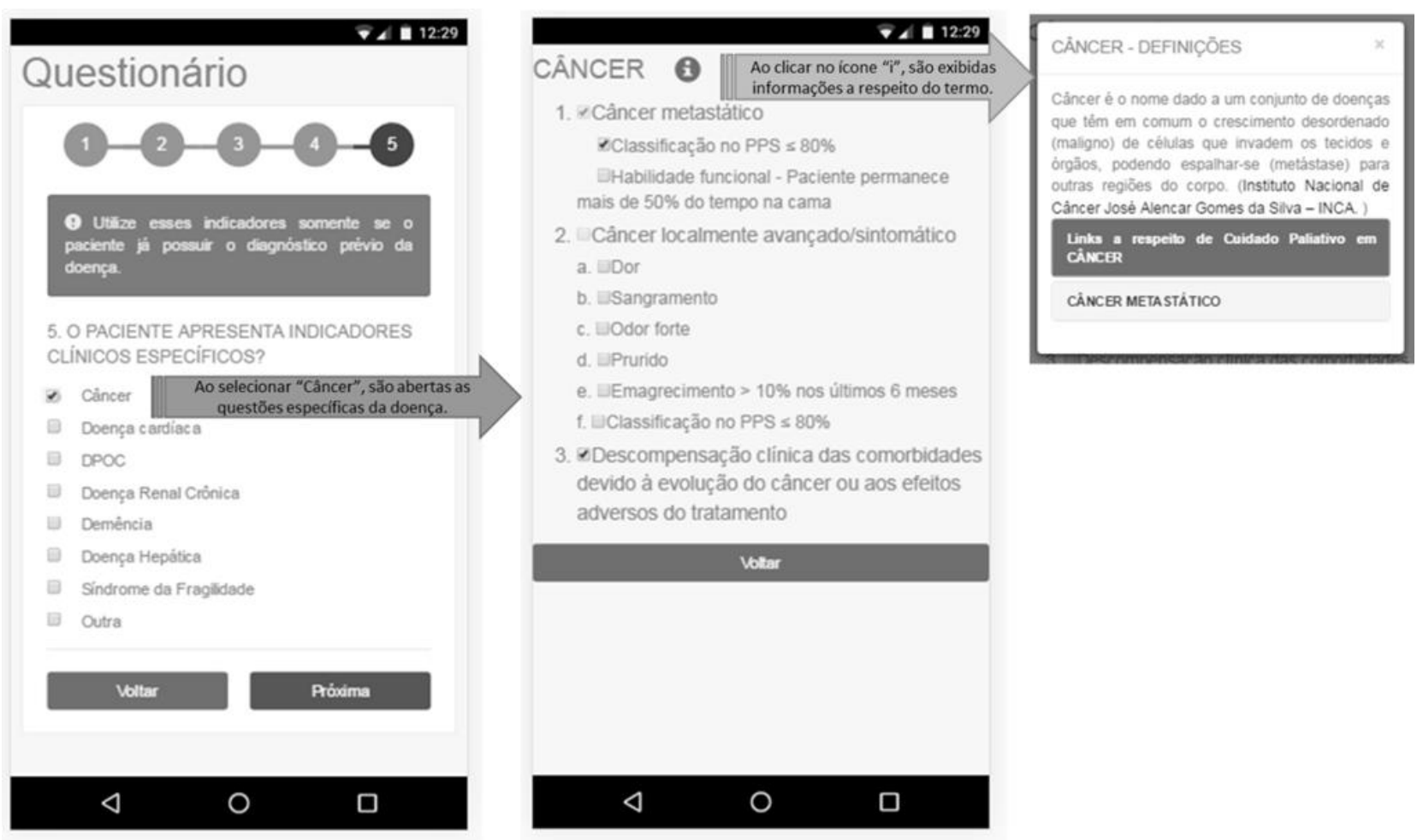

Figura 4. Protótipo do questionário “Quem precisa de Cuidados Paliativos?”

As respostas têm formato múltipla escolha e guiam os profissionais ao longo do preenchimento. Novas perguntas são exibidas à medida que o usuário avança as etapas do questionário até a sua finalização. $O$ resultado final será mediante indicação ou não dos Cuidados Paliativos, conforme Tabela 2.

Tabela 2. Mensagens da funcionalidade "Quem precisa de cuidados paliativos?"

Cuidados paliativos são indicados
Seu paciente tem indicação de iniciar os Cuidados
Paliativos.
Valor do PPS: xxx
Existem sintomas que prejudicam a qualidade de vida
do paciente. Priorize manejá-los.
(São exibidas orientações sobre cada sinal/sintoma do
paciente, informados ao longo do questionário)

\section{Cuidados paliativos não são indicados}

Seu paciente ainda não tem indicação de iniciar os Cuidados Paliativos.

Sugerimos que você continue o acompanhamento e reavalie periodicamente o paciente.

\section{Discussão}

A utilização de aparelhos móveis por profissionais de saúde é bastante elevada, atingindo até $85 \%$ dos profissionais, segundo revisão de Garritty e colaboradores (Garritty \& El Emam, 2006).

Estudos robustos evidenciam que profissionais com acesso à educação continuada ou a ferramentas de referência, podem prestar melhor assistência à saúde (Whittaker et al., 2016).

Em 2 de junho de 2017, o aplicativo possui a nota 4,8 (sendo 5,0 a nota máxima) no Google Play e mais de 2.400 downloads, número que pode ser ampliado com a implementação de novas funcionalidades. Além disso, 
atualmente possui destaque entre os aplicativos ligados a área de saúde e é o primeiro lugar nas pesquisas sobre aplicativos para Cuidados Paliativos ${ }^{4}$.

A boa adesão ao aplicativo pode ser associada à facilidade de uso e pelo suporte às melhores práticas dos Cuidados Paliativos dentro e fora do ambiente hospitalar ${ }^{5}$. Entretanto, a sua usabilidade, do ponto de vista dos usuários, será avaliada em um trabalho futuro.

\section{Conclusão}

O aplicativo "Cuidados Paliativos" é um instrumento que pode auxiliar os profissionais da área de saúde a avaliarem de maneira prática e rápida o controle dos sintomas e da condição funcional do paciente por meio das escalas ESAS e PPS. Este aplicativo também pode apoiar profissionais de saúde na comunicação de más notícias, tarefa rotineira neste contexto, mas que exige habilidade e treinamento específico.

Ao auxiliar no reconhecimento dos sinais e sintomas apresentados pelos pacientes portadores de doença ameaçadora da continuidade da vida e capacitar o profissional de saúde a comunicar notícias ruins, o aplicativo tem o potencial de promover alívio ao sofrimento e agregar qualidade à vida e ao processo de morrer.

A expansão do conteúdo está programada para aumentar a utilidade do aplicativo, tendo como objetivo a inserção de funcionalidades para o próprio paciente, assim como o registro de seus dados para o monitoramento e visualização da progressão do seu quadro clínico dentro do conceito atual de empoderamento da saúde.

\section{Referências}

ANCP BRASIL. (2009). Manual de Cuidados Paliativos. Academia Nacional de Cuidados Paliativos. doi:10.1157/13074624.

Clayer, M. T. (2007). Clinical Practice Guidelines for Communicating Prognosis and End-of-Life Issues with Adults in the Advanced Stages of a Life-Limiting Illness, and Their Caregivers. Medical Journal of Australia, 187(8), 478. doi:cla11246_fm [pii].

Ferreira, D., Costa, F., Alonso, F., Alves, P., \& Nunes, T. (2005). Um Modelo Ágil Para Gestão de Projectos de Software. Faculdade de Engenharia - Universidade do Porto. Retrieved from http://paginas.fe.up.pt/ aaguiar/es/artigos finais/es_final_19.pdf

Garritty, C., \& Emam, K. (2006). Who's Using PDAs? Estimates of PDA Use by Health Care Providers: A Systematic Review of Surveys. Journal of Medical Internet Research, 8(2): e7. doi:10.2196/jmir.8.2.e7.

Gonçalves, E., \& Bonifácio, B. (2014). Avaliação do impacto do Contexto e do canal de comunicação na usabilidade de aplicativos móveis. In IHC '14 Companion Proceedings of the 13th Brazilian Symposium on Human Factors in Computing Systems (pp. 51-52). Foz do Iguaçu, Brasil: Sociedade Brasileira de Computação Porto Alegre. Retrieved from http://dl.acm.org/citation.cfm?id=2738184

Google Inc. (n.d.) Apps Para Android No Google Play. Retrieved from https://play.google.com/store/apps?hl=pt

Google Inc. (n.d.) Termos de Serviço Do Google Play. Retrieved from https://play.google.com/intl/ptbr_br/about/play-terms.html

Hospital das Clínicas da UFMG. (2017). Aplicativo Do HC-UFMG Sobre Cuidados Paliativos É Um Dos Mais Baixados Do Brasil. Retrieved from http://www.enf.ufmg.br/index.php/ noticias/426-aplicativo-do-hc-ufmg-sobre-cuidadospaliativos-e-um-dos-mais-baixados-do-brasil/

International Organization for Standardization (2011). ISO/IEC 25010 - Systems and Software Engineering -- SQuaRE Software Product Quality Requirements and Evaluation -- System and Software Quality Models.

\footnotetext{
${ }^{4}$ Google Inc., "Apps Para Android No Google Play."

${ }^{5}$ Hospital das Clínicas da UFMG, "Aplicativo Do HC-UFMG Sobre Cuidados Paliativos É Um Dos Mais Baixados Do Brasil."
} 
Maciel, M., \& Carvalho, R. (2009). Palliative Performance Scale PPS: Versão 2. Tradução Brasileira Para a Língua Portuguesa [Internet]. São Paulo.

Maciel, M. (2008). Definições e Princípios. Cuidado Paliativo, 15.

Maida, V., et al. (2008). Correlation between Braden Scale and Palliative Performance Scale in Advanced Illness. International Wound Journal, 5(4), 585-590. doi:10.1111/j.1742-481X.2008.00475.x.

Monteiro, D. (2012). Tradução e adaptação transcultural do instrumento edmonton symptom assessment system para uso em cuidados paliativos tt - Translation and cultural adaptation of the instrument edmonton symptom assessment system for use in palliative care. Revista Gaúcha de Enfermagem, 34(2), 80. Retrieved from http://www.bibliotecadigital.ufrgs.br/da.php?nrb=000850873\&loc=2012\&l=54601f0278c9629f.

Neto, I. (2006). Modelos de Controlo de Sintomas. Manual de Cuidados Paliativos. Lisboa: Núcleo de Cuidados Paliativos, Centro de Bioética, Faculdade de Medicina de Lisboa.

Prezotto, E., \& Boniati, B. (2014). Estudo de Frameworks Multiplataforma Para Desenvolvimento de Aplicações Mobile Híbridas. 72-79. Retrieved from http://www.eati.info/eati/2014/assets/anais/artigo8.pdf

Rivero, L., \& Conte, T. (2014). Aplicando design e avaliação de usabilidade para melhorar a qualidade de um aplicativo web móvel. Anais Do Simpósio Brasileiro de Qualidade de Software (SBQS 2014), 25010, $247-254$.

Thomas, K. (2011). Prognostic Indicator Guidance (PIG). The Gold Standards Framework Centre In End of Life Care $\mathrm{CIC}$.

Whittaker, R., et al. (2016). Mobile Phone-Based Interventions for Smoking Cessation. Cochrane Database of Systematic Reviews 4(4). doi:10.1002/14651858.CD006611.pub4

\section{Sobre os Autores}

Thábata Queiroz Vivas de Sá é analista de sistemas no Centro de Telessáude do Hospital das Clínicas da Universidade Federal de Minas Gerais e especialista em Bancos de Dados e Informática em Saúde.

Paulo Rodrigues Gomes é analista de sistemas no Centro de Telessáude do Hospital das Clínicas da Universidade Federal de Minas Gerais.

Breno Max Horta Melo é analista de sistemas no Centro de Telessáude do Hospital das Clínicas da Universidade Federal de Minas Gerais.

Taís Marina de Souza é médica e pesquisadora no Centro de Telessaúde do Hospital das Clínicas da Universidade Federal de Minas Gerais.

Laila Carine Ferreira Lodi Junqueira é médica especialista em Clínica Médica e Geriatria e pesquisadora no Centro de Telessaúde do Hospital das Clínicas da Universidade Federal de Minas Gerais.

Munir Murad Junior (MsC) é médico especialista em Clínica Médica e Oncologia e mestre em Ciências da Saúde, sendo coordenador da oncologia do Hospital das Clínicas da Universidade Federal de Minas Gerais.

Antônio Luiz Pinho Ribeiro (PhD) é médico especialista em Clínica Médica e Cardiologia e professor Titular do Departamento de Clínica Médica da Faculdade de Medicina da Universidade Federal de Minas Gerais. Doutor em Medicina e Coordenador da Rede de Teleassistência de Minas Gerais e do Centro de Telessaúde do Hospital das Clínicas da Universidade Federal de Minas Gerais. 